\title{
Passivity-Based Asynchronous Control for Markov Jump Systems
}

\author{
Zheng-Guang $\mathrm{Wu}$, Peng Shi, Zhan Shu, Hongye Su, and Renquan $\mathrm{Lu}$
}

\begin{abstract}
This paper studies the problem of passivity-based asynchronous control for discrete-time Markov jump systems. The asynchronization phenomenon appears between the system modes and controller modes, which is described by a hidden Markov model. Accordingly, the resultant closed-loop system is named as a hidden Markov jump system. By utilizing the matrix inequality technique, three equivalent sufficient conditions are proposed to ensure the stochastic passivity of the hidden Markov jump systems. Based on the established conditions, the design of asynchronous controller, which covers synchronous controller and mode-independent controller as special cases, is addressed. A numerical example is given to demonstrate the effectiveness of the derived results.
\end{abstract}

Index Terms-Markov jump systems, switching, hidden Markov model, asynchronous control, passivity

\section{INTRODUCTION}

In the past few decades, significant progress has been made on the theory of Markov jump systems, which is one of the most active research areas in systems and control. The reason is mainly that Markov jump systems are suitable mathematical models to represent various practical systems whose structure is subject to random abrupt variation mainly due to, for example, changing in subsystem interconnections, random component failures or repairs, sudden environmental changes, and uncontrolled configuration changes. As a result, fruitful results have been reported in this field. For example, much progress has been made in the study of the stability and stabilization problems for Markov jump systems; see, e.g., [1]-[6] and the references therein. When both the Markov jump parameters and time delays appear in the systems, the problems of stability and stabilization have been studied in [7]-[9]. The problems of $H_{\infty}$ control and sliding mode control

This work was partially supported by the Australian Research Council (DP140102180, LP140100471), the National High Technology Research and Development Program of China (863 Program 2012AA041703), the National Natural Science Foundation of China $(61573112,61304072)$, the China National Funds for Distinguished Young Scientists (61425009), and the Zhejiang Provincial Natural Science Foundation of China (LR16F030001).

Z.-G. Wu and H. Su are with the National Laboratory of Industrial Control Technology, Institute of Cyber-Systems and Control, Zhejiang University, Yuquan Campus, Hangzhou Zhejiang, 310027, PR China (e-mail: nashwzhg@gmail.com;hysu@iipc.zju.edu.cn).

P. Shi is with the School of Electrical and Electronic Engineering, The University of Adelaide, SA 5005, Australia. He is also with the College of Engineering and Science, Victoria University, Melbourne, VIC 8001, Australia; and College of Automation, Harbin Engineering University, Harbin, 150001, China (e-mail: peng.shi@vu.edu.au).

Z. Shu is with the School of Engineering Sciences, University of Southampton, Southampton SO17 1BJ, U.K. (e-mail: z.shu@ soton.ac.uk).

R. Lu is with with School of Automation, Guangdong University of Technology, and Guangdong Key Laboratory of IoT Information Processing, Guangzhou 510006, China (e-mail: rqlu@hdu.edu.cn). have been investigated for Markov jump systems in [10] and [11], respectively. In [12], the robust $H_{\infty}$ filtering problem has been addressed for discrete-time Markov jump systems subject to both randomly occurring nonlinearities and sensor saturation. When time delays appear in the Markov jump systems, the $H_{\infty}$ and $l_{2}-l_{\infty}$ filtering problems have been considered in [13] and [14], respectively.

It should be pointed out that most existing results on control and filtering of Markov jump systems are based on an common and important assumption that the mode information of plant is fully available to the controller/filter at every instant of time to ensure the switching of controller/filter synchronous with that of plant. Accordingly, the designed controller/filter is named as mode-dependent or synchronous controller/filter. However, such an ideal assumption cannot always be satisfied in real world applications. For example, in networked control systems, network-induced delays and packet loss inevitably make the mode information of plant not completely accessible, which leads to the asynchronization phenomenon between system modes and controller/filter modes. Thus, it is important and necessary to study the asynchronous control/filtering for Markov jump systems. The stabilization problem for discretetime Markov jump systems with time delay in the mode signal has been investigated in [15] and a sufficient condition has been proposed for the design of a controller with delayed mode information such that the closed-loop system is stochastically stable. The stabilization problem of discrete-time Markov jump systems with a non-accessible jumping parameter has been studied in [16], where the linear matrix inequality (LMI) method has been proposed for designing a mode-independent controller, which can be regarded as a special asynchronous controller. In [17], [18], some approaches have been proposed to design the mode-independent $H_{\infty}$ filter for continuous-time Markov jump systems. Very recently, the problem of asynchronous $l_{2}-l_{\infty}$ filtering for discrete-time stochastic Markov jump systems has been addressed in [19], where the existence criterion of the desired asynchronous filter with piecewise homogeneous Markov chain has been proposed. Although the importance of asynchronous control/filtering for Markov jump systems has been widely recognized, the related problems have not been fully addressed, which is the motivation for the current work.

In this paper, the problem of stochastically passive asynchronous control for discrete-time Markov jump systems is studied. The system mode is assumed to be asynchronous with the controller mode, which is caused by various constraints on signal processing and transmission in practice. A hidden Markov model is introduced to describe the asynchroniza- 
tion phenomenon. As a result, the corresponding closed-loop system is constructed as a hidden Markov jump system. By adopting the matrix inequality approach, several criteria are given to ensure that the hidden Markov jump system is stochastically passive based on one of which the design method of the desired asynchronous controller is proposed. A numerical example is used to show the effectiveness of the theoretic results obtained.

Notation: The notations used throughout this paper are fairly standard. $\mathbb{R}^{n}$ and $\mathbb{R}^{m \times n}$ denote the $n$-dimensional Euclidean space and the set of all $m \times n$ real matrices, respectively. The notation $X>Y(X \geqslant Y)$, where $X$ and $Y$ are symmetric matrices, means that $X-Y$ is positive definite (positive semidefinite). $\operatorname{diag}\{\ldots\}$ stands for a block-diagonal matrix and $\|\cdot\|$ denotes the Euclidean norm of a vector and its induced norm of a matrix. $(\Omega, \mathcal{F}, \mathcal{P})$ is a probability space, $\Omega$ is the sample space, $\mathcal{F}$ is the $\sigma$-algebra of subsets of the sample space and $\mathcal{P}$ is the probability measure on $\mathcal{F} . \mathscr{E}\{\cdot\}$ denotes the expectation operator with respect to some probability measure $\mathcal{P} . l_{2}[0,+\infty)$ is the space of square summable infinite sequence.

\section{Preliminaries}

Fix a probability space $(\Omega, \mathcal{F}, \mathcal{P})$ and consider the following discrete-time Markov jump system:

$$
\left\{\begin{aligned}
x(k+1) & =A(r(k)) x(k)+D(r(k)) u(k)+B(r(k)) v(k), \\
z(k) & =C(r(k)) x(k)+E(r(k)) u(k)+L(r(k)) v(k),
\end{aligned}\right.
$$

where $x(k) \in \mathbb{R}^{n}$ is the system state, $u(k) \in \mathbb{R}^{m}$ is the control input, $z(k) \in \mathbb{R}^{q}$ is the controlled output, and $v(k) \in \mathbb{R}^{p}$ is the disturbance input that belongs to $l_{2}[0, \infty)$. $A(r(k)), D(r(k)), B(r(k)), C(r(k)), E(r(k))$ and $L(r(k))$ are known real constant matrices with appropriate dimensions. The parameter $\{r(k), k \geqslant 0\}$ represents a Markov chain taking values in a finite set $\mathscr{N}=\{1,2, \cdots, N\}$ with transition probability matrix $\Pi=\left\{\pi_{i j}\right\}$ given by

$$
\operatorname{Pr}\{r(k+1)=j \mid r(k)=i\}=\pi_{i j},
$$

where $0 \leqslant \pi_{i j} \leqslant 1$ for all $i, j \in \mathscr{N}$, and $\sum_{j=1}^{N} \pi_{i j}=1$ for all $i \in \mathscr{N}$.

In this paper, we consider the following controller:

$$
u(k)=K(\sigma(k)) x(k),
$$

where $K(\sigma(k))$ is a controller gain matrix to be determined, and the parameter $\{\sigma(k), k \geqslant 0\}$ takes values in a finite set $\mathscr{M}=\{1,2, \cdots, M\}$ with conditional probability matrix $\Omega=$ $\left\{\mu_{i \phi}\right\}$ given by:

$$
\operatorname{Pr}\{\sigma(k)=\phi \mid r(k)=i\}=\mu_{i \phi},
$$

where $0 \leqslant \mu_{i \phi} \leqslant 1$ for all $i \in \mathscr{N}$ and $\phi \in \mathscr{M}$, and $\sum_{\phi=1}^{M} \mu_{i \phi}=1$ for all $i \in \mathscr{N}$.

Remark 1: In practice, the information of system modes accessible to controller is often inaccurate, that is, the actual modes of system are hidden to the controller, which leads to the fact that the controller modes that can be regarded as the observation value of system modes do not synchronize with systems modes. In this paper, we introduce stochastic variable $\sigma(k)$ to present the modes of controller, which is different from the Markov chain $r(k)$, but depends on it subject to the conditional probability (4). It can be found that the set $(r(k), \sigma(k), \Pi, \Omega)$ constructs a hidden Markov model [20]. To the best of authors' knowledge, our work is the first to make use of the hidden Markov model to describe the non-synchronization phenomenon between system modes and controller modes.

Remark 2: It is noted that when $\mathscr{N}=\mathscr{M}$ and $\mu_{i \phi}=1$ for $i=\phi$, the controller (3) becomes a synchronous controller, and when $\mathscr{M}=\{1\}$, that is, the controller (3) only has one mode, the asynchronous controller (3) reduces to the mode-independent controller. Thus, the asynchronous controller given in this paper covers synchronous controller and mode-independent controller as special cases.

Applying the asynchronous controller (3) to system (1) leads to the following closed-loop system,

$$
\left\{\begin{aligned}
x(k+1)= & (A(r(k))+D(r(k)) K(\sigma(k))) x(k) \\
& +B(r(k)) v(k), \\
z(k)= & (C(r(k))+E(r(k)) K(\sigma(k))) x(k) \\
& +L(r(k)) v(k) .
\end{aligned}\right.
$$

Remark 3: It is worth mentioning that in this paper, the closed-loop system (5) is named as hidden Markov jump system due to the fact that the hidden Markov model $(r(k), \sigma(k), \Pi, \Omega)$ is included in the system. Up to now, few results have been proposed for such kind of system.

In this paper, the following definition will be used.

Definition 1: System (5) is said to be stochastically passive if there exists a scalar $\gamma>0$ such that for all $k_{0} \geqslant 0$,

$$
2 \sum_{s=0}^{k_{0}} \mathscr{E}\left\{z(s)^{\mathrm{T}} v(s)\right\} \geqslant-\gamma \sum_{s=0}^{k_{0}}\|v(s)\|^{2}
$$

under the zero initial condition.

The objective of this paper is to design an asynchronous controller (3) for system (1) such that system (5) is stochastically passive.

For notational simplicity, in the sequel, for each possible $r(k)=i \in \mathscr{N}$ and $\sigma(k)=\phi \in \mathscr{M}$, the matrices $M(r(k))$ and $N(\sigma(k))$ will be denoted by $M_{i}$ and $N_{\phi}$, respectively.

\section{MAIN RESUltS}

In this section, the deign method of asynchronous controller will be given for system (1). Firstly, we will present some conditions to ensure system (5) is stochastically passive.

Theorem 1: System (5) is stochastically passive, if there exist matrices $P_{i}>0$ and a scalar $\gamma>0$ such that for any $i \in \mathscr{N}$ and $\phi \in \mathscr{M}$,

$$
\begin{aligned}
\mathscr{Z}_{i} & =\sum_{\phi=1}^{M} \mu_{i \phi}\left(\hat{A}_{i \phi}^{\mathrm{T}} X_{i} \hat{A}_{i \phi}-\mathscr{H}_{i \phi} \mathscr{S}_{i}^{-1} \mathscr{H}_{i \phi}^{\mathrm{T}}\right)-P_{i}<0, \\
\mathscr{S}_{i} & =B_{i}^{\mathrm{T}} X_{i} B_{i}-L_{i}^{\mathrm{T}}-L_{i}-\gamma I<0,
\end{aligned}
$$

where

$$
X_{i}=\sum_{j=1}^{N} \pi_{i j} P_{j}, \mathscr{H}_{i \phi}=\hat{A}_{i \phi}^{\mathrm{T}} X_{i} B_{i}-\hat{C}_{i \phi}^{\mathrm{T}},
$$




$$
\hat{A}_{i \phi}=A_{i}+D_{i} K_{\phi}, \hat{C}_{i \phi}=C_{i}+E_{i} K_{\phi}
$$

Proof: Consider the following Lyapunov functional for system (5):

$$
V(k, x(k), r(k))=x(k)^{\mathrm{T}} P(r(k)) x(k) .
$$

Letting $\mathscr{E}\{\Delta V(k)\}=\mathscr{E}\{V(k+1, x(k+1), r(k+1)=$ $j) \mid x(k), r(k)=i\}-V(k, x(k), i)$, we have

$$
\mathscr{E}\{\Delta V(k)\}=\mathscr{E}\left\{x(k+1)^{\mathrm{T}} X_{i} x(k+1)\right\}-x(k)^{\mathrm{T}} P_{i} x(k) .
$$

On the other hand, it can be calculated that along the solution of system (5),

$$
\begin{aligned}
\mathscr{E}\left\{x(k+1)^{\mathrm{T}} X_{i} x(k+1)\right\} & \\
= & \sum_{\phi=1}^{M} \mu_{i \phi} \eta(k)^{\mathrm{T}} \mathscr{Y}_{i \phi}^{\mathrm{T}} X_{i} \mathscr{Y}_{i \phi} \eta(k) \\
& =\eta(k)^{\mathrm{T}}\left(\sum_{\phi=1}^{M} \mu_{i \phi} \mathscr{Y}_{i \phi}^{\mathrm{T}} X_{i} \mathscr{Y}_{i \phi}\right) \eta(k),
\end{aligned}
$$

where

$$
\mathscr{Y}_{i \phi}=\left[\begin{array}{c}
\hat{A}_{i \phi}^{\mathrm{T}} \\
B_{i}^{\mathrm{T}}
\end{array}\right]^{\mathrm{T}}, \eta(k)=\left[\begin{array}{l}
x(k) \\
v(k)
\end{array}\right] .
$$

From (10) and (11), we get that

$$
\begin{aligned}
\mathscr{E}\{ & \Delta V(k)\} \\
& =\eta(k)^{\mathrm{T}}\left(\sum_{\phi=1}^{M} \mu_{i \phi} \mathscr{Y}_{i \phi}^{\mathrm{T}} X_{i} \mathscr{Y}_{i \phi}\right) \eta(k)-x(k)^{\mathrm{T}} P_{i} x(k),
\end{aligned}
$$

It is noted that

$$
\begin{aligned}
\mathscr{E}\left\{z(k)^{\mathrm{T}} v(k)\right\} & =\sum_{\phi=1}^{M} \mu_{i \phi} \eta(k)^{\mathrm{T}} \mathscr{U}_{i \phi}^{\mathrm{T}} v(k) \\
& =\eta(k)^{\mathrm{T}}\left(\sum_{\phi=1}^{M} \mu_{i \phi} \mathscr{U}_{i \phi}^{\mathrm{T}}\right) v(k),
\end{aligned}
$$

where

$$
\mathscr{U}_{i \phi}=\left[\begin{array}{c}
\hat{C}_{i \phi}^{\mathrm{T}} \\
L_{i}^{\mathrm{T}}
\end{array}\right]^{\mathrm{T}}
$$

Thus, we obtain from (12) and (13) that under the zero initial condition

$$
\begin{aligned}
J_{z v} \triangleq & \sum_{s=0}^{k_{0}} \mathscr{E}\left\{-2 z(s)^{\mathrm{T}} v(s)-\gamma v(s)^{\mathrm{T}} v(s)\right\} \\
\leqslant & \sum_{s=0}^{k_{0}} \mathscr{E}\left\{\Delta V(s)-2 z(s)^{\mathrm{T}} v(s)-\gamma v(s)^{\mathrm{T}} v(s)\right\} \\
= & \sum_{s=0}^{k_{0}} \eta(s)^{\mathrm{T}}\left(\sum_{\phi=1}^{M} \mu_{i \phi} \mathscr{Y}_{i \phi}^{\mathrm{T}} X_{i} \mathscr{Y}_{i \phi}\right) \eta(s) \\
& +\sum_{s=0}^{k_{0}}\left(-2 \eta(s)^{\mathrm{T}}\left(\sum_{\phi=1}^{M} \mu_{i \phi} \mathscr{U}_{i \phi}^{\mathrm{T}}\right) v(s)\right)
\end{aligned}
$$

$$
\begin{aligned}
& +\sum_{s=0}^{k_{0}}\left(-x(s)^{\mathrm{T}} P_{i} x(k)-\gamma v(k)^{\mathrm{T}} v(s)\right) \\
= & \sum_{s=0}^{k_{0}}\left(v(s)^{\mathrm{T}}\left(\sum_{\phi=1}^{M} \mu_{i \phi} \Xi_{i \phi}\right) v(s)-x(s)^{\mathrm{T}} P_{i} x(s)\right),
\end{aligned}
$$

where

$$
\Xi_{i \phi}=\left[\begin{array}{cc}
\hat{A}_{i \phi}^{\mathrm{T}} X_{i} \hat{A}_{i \phi} & \mathscr{H}_{i \phi} \\
* & \mathscr{S}_{i}
\end{array}\right]
$$

Furthermore, based on Schur complement, it can be found that

$$
\left[\begin{array}{cc}
\mathscr{H}_{i \phi} \mathscr{S}_{i}^{-1} \mathscr{H}_{i \phi}^{\mathrm{T}} & \mathscr{H}_{i \phi} \\
* & \mathscr{S}_{i}
\end{array}\right] \leqslant 0
$$

which implies

$$
\Xi_{i \phi} \leqslant\left[\begin{array}{cc}
\hat{A}_{i \phi}^{\mathrm{T}} X_{i} \hat{A}_{i \phi}-\mathscr{H}_{i \phi} \mathscr{S}_{i}^{-1} \mathscr{H}_{i \phi}^{\mathrm{T}} & 0 \\
* & 0
\end{array}\right]
$$

Thus,

$$
\sum_{\phi=1}^{M} \mu_{i \phi} \Xi_{i \phi} \leqslant \sum_{\phi=1}^{M} \mu_{i \phi}\left[\begin{array}{cc}
\hat{A}_{i \phi}^{\mathrm{T}} X_{i} \hat{A}_{i \phi}-\mathscr{H}_{i \phi} \mathscr{S}_{i}^{-1} \mathscr{H}_{i \phi}^{\mathrm{T}} & 0 \\
* & 0
\end{array}\right]
$$

Applying the above inequality to (14) yields that

$$
J_{z v} \leqslant \sum_{s=0}^{k_{0}} x(s)^{\mathrm{T}} \mathscr{Z}_{i} x(s) .
$$

Therefore, it follows (5) that

$$
J_{z v} \leqslant 0,
$$

which implies that (6) holds for all $k_{0} \geqslant 0$. This completes the proof.

Note that in Theorem 1, a passivity condition is proposed for system (5). However, the criterion cannot be directly applied to design the desired asynchronous controller (3) due to its complicated structure. To overcome this difficulty, some equivalent conditions will be given in the following theorem.

Theorem 2: The following three assertions are equivalent:

(i) There exist matrices $P_{i}>0$ and a scalar $\gamma>0$ such that (6) holds;

(ii) There exist matrices $P_{i}>0$ and $R_{i \phi}>0$ and a scalar $\gamma>0$ such that

$$
\begin{aligned}
& \sum_{\phi=1}^{M} \mu_{i \phi} R_{i \phi}<P_{i}, \\
& {\left[\begin{array}{ccc}
-R_{i \phi} & -\left(C_{i}+E_{i} K_{\phi}\right)^{\mathrm{T}} & \mathscr{Q}_{i \phi} \\
* & -L_{i}^{\mathrm{T}}-L_{i}-\gamma I & \mathscr{D}_{i} \\
* & * & \mathscr{Y}_{i}
\end{array}\right]<0,}
\end{aligned}
$$

where

$$
\mathscr{Q}_{i \phi}=\left[\begin{array}{c}
\sqrt{\pi_{i 1}} P_{1}\left(A_{i}+D_{i} K_{\phi}\right) \\
\sqrt{\pi_{i 2}} P_{2}\left(A_{i}+D_{i} K_{\phi}\right) \\
\vdots \\
\sqrt{\pi_{i N}} P_{N}\left(A_{i}+D_{i} K_{\phi}\right)
\end{array}\right]^{\mathrm{T}}, \mathscr{D}_{i}=\left[\begin{array}{c}
\sqrt{\pi_{i 1}} P_{1} B_{i} \\
\sqrt{\pi_{i 2}} P_{2} B_{i} \\
\vdots \\
\sqrt{\pi_{i N}} P_{N} B_{i}
\end{array}\right]^{\mathrm{T}},
$$




$$
\mathscr{Y}_{i}=\operatorname{diag}\left\{-P_{1},-P_{2}, \cdots,-P_{N}\right\} .
$$

(iii) There exist matrices $\bar{P}_{i}>0$ and $\bar{R}_{i \phi}>0$ and a scalar $\gamma>0$ such that

$$
\begin{aligned}
& {\left[\begin{array}{cc}
-\bar{P}_{i} & \overline{\mathscr{R}}_{i} \\
* & \overline{\mathscr{X}}_{i}
\end{array}\right]<0,} \\
& {\left[\begin{array}{ccc}
-\bar{R}_{i \phi} & -\bar{R}_{i \phi}\left(C_{i}+E_{i} K_{\phi}\right)^{\mathrm{T}} & \overline{\mathscr{Q}}_{i \phi} \\
* & -L_{i}^{\mathrm{T}}-L_{i}-\gamma I & \overline{\mathscr{D}}_{i} \\
* & * & \overline{\mathscr{Y}}_{i}
\end{array}\right]<0,}
\end{aligned}
$$

where

$$
\begin{aligned}
& \overline{\mathscr{R}}_{i}=\left[\begin{array}{llll}
\sqrt{\mu_{i 1}} \bar{P}_{i} & \sqrt{\mu_{i 2}} \bar{P}_{i} & \cdots & \sqrt{\mu_{i M}} \bar{P}_{i}
\end{array}\right], \\
& \overline{\mathscr{X}}_{i}=\operatorname{diag}\left\{-\bar{R}_{i 1},-\bar{R}_{i 2}, \cdots,-\bar{R}_{i M}\right\} \text {, } \\
& \overline{\mathscr{Q}}_{i \phi}=\left[\begin{array}{c}
\sqrt{\pi_{i 1}}\left(A_{i}+D_{i} K_{\phi}\right) \bar{R}_{i \phi} \\
\sqrt{\pi_{i 2}}\left(A_{i}+D_{i} K_{\phi}\right) \bar{R}_{i \phi} \\
\vdots \\
\sqrt{\pi_{i N}}\left(A_{i}+D_{i} K_{\phi}\right) \bar{R}_{i \phi}
\end{array}\right]^{\mathrm{T}}, \overline{\mathscr{D}}_{i}=\left[\begin{array}{c}
\sqrt{\pi_{i 1}} B_{i} \\
\sqrt{\pi_{i 2}} B_{i} \\
\vdots \\
\sqrt{\pi_{i N}} B_{i}
\end{array}\right]^{\mathrm{T}}, \\
& \overline{\mathscr{Y}}_{i}=\operatorname{diag}\left\{-\bar{P}_{1},-\bar{P}_{2}, \cdots,-\bar{P}_{N}\right\} \text {. }
\end{aligned}
$$

Proof: (i) $\rightarrow$ (ii)

If there exist matrices $P_{i}>0$ and a scalar $\gamma>0$ such that (6) holds, then there must exist a scalar $\varepsilon>0$ such that

$$
\sum_{\phi=1}^{M} \mu_{i \phi}\left(\hat{A}_{i \phi}^{\mathrm{T}} X_{i} \hat{A}_{i \phi}-\mathscr{H}_{i \phi} \mathscr{S}_{i}^{-1} \mathscr{H}_{i \phi}^{\mathrm{T}}+\varepsilon I\right)-P_{i}<0 .
$$

Choose

$$
R_{i \phi}=\hat{A}_{i \phi}^{\mathrm{T}} X_{i} \hat{A}_{i \phi}-\mathscr{H}_{i \phi} \mathscr{S}_{i}^{-1} \mathscr{H}_{i \phi}^{\mathrm{T}}+\varepsilon I,
$$

which implies (20) holds and

$$
R_{i \phi}-\hat{A}_{i \phi}^{\mathrm{T}} X_{i} \hat{A}_{i \phi}+\mathscr{H}_{i \phi} \mathscr{S}_{i}^{-1} \mathscr{H}_{i \phi}^{\mathrm{T}}>0 .
$$

Applying Schur complement, we can get (21).

$$
\text { (ii) } \rightarrow \text { (i) }
$$

If there exist matrices $P_{i}>0$ and a scalar $\gamma>0$ such that (20) and (21) holds, then based on Schur complement, we get (29) hold. Thus

$$
\sum_{\phi=1}^{M} \mu_{i \phi}\left(\hat{A}_{i \phi}^{\mathrm{T}} X_{i} \hat{A}_{i \phi}-\mathscr{H}_{i \phi} \mathscr{S}_{i}^{-1} \mathscr{H}_{i \phi}^{\mathrm{T}}\right)-\sum_{\phi=1}^{M} \mu_{i \phi} R_{i \phi}<0 .
$$

Using (20) and (27), we can get (6).

(ii) $\leftrightarrow$ (iii)

It can be found from Schur complement that (20) is equivalent to

$$
\left[\begin{array}{cc}
-P_{i} & \mathscr{J}_{i} \\
* & \bar{X}_{i}
\end{array}\right]<0
$$

where $\quad \bar{R}_{i \phi} \quad=\quad R_{i \phi}^{-1}$ and $\mathscr{J}_{i}=$ $\left[\begin{array}{llll}\sqrt{\mu_{i 1}} I & \sqrt{\mu_{i 2}} I & \cdots & \sqrt{\mu_{i M}} I\end{array}\right]$. Denoting $\bar{P}_{i}=P_{i}^{-1}$, and pre- and post-multiplying (28) by $\operatorname{diag}\{-\bar{P}_{i}, \underbrace{I, I, \cdots, I}_{M}\}$, respectively, we can show (28) is equivalent to (22). On the other hand, pre- and post-multiplying (21) by
$\operatorname{diag}\left\{\bar{R}_{i \phi}, I, \bar{P}_{1}, \bar{P}_{2}, \cdots, \bar{P}_{N}\right\}$, respectively, we have (23) is equivalent to (21). This completes the proof.

Remark 4: It is noted that two novel passivity conditions equivalent to the one in Theorem 1 are obtained in Theorem 2. The advantage of (20) and (21) lies in the separation of $\mu_{i \phi}$ and $K_{\phi}$. While in (22) and (23), not only $\mu_{i \phi}$ and $K_{\phi}$ are separated, but also $K_{\phi}$ and $P_{i}$ are separated. Thus, from control design point view, the assertion (iii), which is more flexible to parametrize $K_{\phi}$ by introducing free parameter matrices, is more desirable than the other two assertions.

Next, we shall make use of assertion (iii) to deal with passivity-based asynchronous control for system (1), and a sufficient condition is obtained for the existence of a asynchronous controller.

Theorem 3: System (5) is stochastically passive, if there exist matrices $\bar{P}_{i}>0, \bar{R}_{i \phi}>0$, matrices $K_{\phi}, G_{\phi}$, and a scalar $\gamma>0$ such that (22) and the following LMIs holds,

$$
\left[\begin{array}{ccc}
\bar{R}_{i \phi}-G_{\phi}^{\mathrm{T}}-G_{\phi} & -\left(C_{i} G_{\phi}+E_{i} \bar{K}_{\phi}\right)^{\mathrm{T}} & \hat{\mathscr{Q}}_{i \phi} \\
* & -L_{i}^{\mathrm{T}}-L_{i}-\gamma I & \overline{\mathscr{D}}_{i} \\
* & * & \overline{\mathscr{Y}}_{i}
\end{array}\right]<0,
$$

where

$$
\hat{\mathscr{Q}}_{i \phi}=\left[\begin{array}{c}
\sqrt{\pi_{i 1}}\left(A_{i} G_{\phi}+D_{i} \bar{K}_{\phi}\right) \\
\sqrt{\pi_{i 2}}\left(A_{i} G_{\phi}+D_{i} \bar{K}_{\phi}\right) \\
\vdots \\
\sqrt{\pi_{i N}}\left(A_{i} G_{\phi}+D_{i} \bar{K}_{\phi}\right)
\end{array}\right]^{\mathrm{T}} .
$$

Furthermore, if (22) and (29) are solvable, a desired asynchronous controller (3) can be chosen with parameter as

$$
K_{\phi}=\bar{K}_{\phi} G_{\phi}^{-1} \text {. }
$$

Proof: Pre- and post-multiplying (23) by $\operatorname{diag}\{G_{\phi}^{\mathrm{T}} \bar{R}_{i \phi}^{-1}, I, \underbrace{I, \cdots, I}_{M}\}$, respectively, yield that

$$
\left[\begin{array}{ccc}
-G_{\phi}^{\mathrm{T}} \bar{R}_{i \phi}^{-1} G_{\phi} & -\left(C_{i} G_{\phi}+E_{i} \bar{K}_{\phi}\right)^{\mathrm{T}} & \hat{\mathscr{Q}}_{i \phi} \\
* & -L_{i}^{\mathrm{T}}-L_{i}-\gamma I & \overline{\mathscr{D}}_{i} \\
* & * & \overline{\mathscr{Y}}_{i}
\end{array}\right]<0 .
$$

On the other hand, noting $\bar{R}_{i \phi}>0$, we have

$$
\begin{aligned}
\bar{R}_{i \phi}-G_{\phi}-G_{\phi}^{\mathrm{T}} & +G_{\phi} \bar{R}_{i \phi}^{-1} G_{\phi}^{\mathrm{T}} \\
& =\left(\bar{R}_{i \phi}-G_{\phi}\right) \bar{R}_{i \phi}^{-1}\left(\bar{R}_{i \phi}-G_{\phi}^{\mathrm{T}}\right) \geqslant 0,
\end{aligned}
$$

which implies $-G_{\phi}^{\mathrm{T}} \bar{R}_{i \phi}^{-1} G_{\phi} \leqslant \bar{R}_{i \phi}-G_{\phi}^{\mathrm{T}}-G_{\phi}$. Combining this with (29), we have that (31) holds. This completes the proof.

Remark 5: It should be pointed out that the design method proposed in Theorem 3 not only can be applied to design the asynchronous controller, but also can be adopted to synchronous controller and mode-independent controller, that is, a unified controller design method is provided for Markov jump system (1).

Remark 6: It is noted that the LMIs in (22) and (29) are not only over the matrix variables, but also over the scalar $\gamma$, which implies that by minimizing $\gamma$ subject to (22) and (29), 
we can obtain the optimal passivity performance $\gamma_{\min }$, and the corresponding asynchronous controller gains as well.

Remark 7: It is worth mentioning that the results given in this paper can be extended easily to dissipativity, which covers passivity as a special case.

\section{NUMERICAL EXAMPLE}

This section will present a numerical example to demonstrate the effectiveness of the method given in the preceding section.

Consider a DC motor device [21], which can be described as system (1) with the following parameters:

$A_{i}=\left[\begin{array}{ccc}a_{11}^{i} & a_{12}^{i} & 0 \\ a_{21}^{i} & a_{22}^{i} & 0 \\ a_{31}^{i} & 0 & a_{33}^{i}\end{array}\right], D_{i}=\left[\begin{array}{c}d_{1}^{i} \\ d_{2}^{i} \\ 0\end{array}\right], B_{i}=b^{i} I, i=1,2,3$

The parameters can be found in Table I.

TABLE I

PARAMETERS OF SyStem (1) REPRESENTING A REAL DC MOTOR DEVICE

\begin{tabular}{c|ccc}
\hline Parameters & $i=1$ & $i=2$ & $i=3$ \\
\hline$a_{11}^{i}$ & -0.4799 & -1.6026 & 0.6346 \\
$a_{12}^{i}$ & 5.1546 & 9.1632 & 0.9178 \\
$a_{21}^{i}$ & -3.8162 & -0.5918 & -0.5056 \\
$a_{22}^{i}$ & 14.4732 & 3.0317 & 2.4811 \\
$a_{31}^{i}$ & 0.1399 & 0.0740 & 0.3865 \\
$a_{33}^{i}$ & -0.9255 & -0.4338 & 0.0982 \\
$d_{1}^{i}$ & 5.8705 & 10.2951 & 0.7874 \\
$d_{2}^{i}$ & 15.5010 & 2.2282 & 1.5302 \\
$b^{i}$ & 0.1 & 0.1 & 0.1 \\
\hline
\end{tabular}

On the other hand, in this example, we choose

$$
C_{i}=\left[\begin{array}{ccc}
1 & 0 & 0 \\
0 & 1.5 & 0 \\
0 & 0 & 2.5
\end{array}\right], E_{i}=\left[\begin{array}{c}
0 \\
0 \\
0.5
\end{array}\right], L_{i}=0
$$

The transition probability matrix

$$
\Pi=\left[\begin{array}{ccc}
0.9 & 0.05 & 0.05 \\
0.36 & 0.6 & 0.04 \\
0.1 & 0.1 & 0.8
\end{array}\right]
$$

and four cases of the conditional probability matrix $\Omega$ are given in Table II.

TABLE II

DIFFERENT CONDITIONAL PROBABILITY MATRICES

\begin{tabular}{c}
\hline Synchronous (Case 1) \\
\begin{tabular}{|c|c|c|c|}
\hline & 1 & 2 & 3 \\
\hline 1 & 1.0 & 0 & 0 \\
\hline 2 & 0 & 1.0 & 0 \\
\hline 3 & 0 & 0 & 1.0 \\
Partially synchronous (Case 3) & \begin{tabular}{|c|c|c|c|c|c|c|}
\hline \\
\hline
\end{tabular} \mid & 2 & 3 \\
\hline 1 & 0.4 & 0.3 & 0.3 \\
\hline 2 & 0.2 & 0.3 & 0.5 \\
\hline 3 & 0 & 0 & 1.0 \\
\hline 1 & 0.4 & 0.3 & 0.3 \\
\hline 2 & 0 & 1.0 & 0 \\
\hline 3 & 0 & 0 & 1.0 \\
\hline
\end{tabular} \\
\begin{tabular}{|l|c|c|c|c|}
\hline & Asynchronous (Case 4) \\
\hline 1 & 1 & 0.4 & 0.3 & 0.3 \\
\hline 2 & 0.2 & 0.3 & 0.5 \\
\hline 3 & 0.4 & 0.5 & 0.1 \\
\hline
\end{tabular}
\end{tabular}

Based on Theorem 3, the optimal passivity performance $\gamma_{\min }$ is obtained in Table III for different conditional probability matrix $\Omega$. It can be seen from Table III that for a higher
TABLE III

OPTIMAL PASSIVITY PERFORMANCE $\gamma_{\min }$ DIFFERENT $\Omega$

\begin{tabular}{llll}
\hline Case 1 & Case 2 & Case 3 & Case 4 \\
\hline 2.5688 & 3.0904 & 3.8323 & 3.9669 \\
\hline
\end{tabular}

mode synchronization rate, the obtained optimal passivity performance $\gamma_{\min }$ is smaller.

As for the asynchronous case (Case 4), by solving the LMIs in (22) and (29), one can get the following controller gain matrices

$$
\begin{aligned}
& K_{1}=\left[\begin{array}{lll}
0.2391 & -0.9086 & -0.0508
\end{array}\right] \\
& K_{2}=\left[\begin{array}{lll}
0.2350 & -0.9046 & -0.0512
\end{array}\right] \\
& K_{3}=\left[\begin{array}{lll}
0.2455 & -0.8813 & -0.0644
\end{array}\right]
\end{aligned}
$$

The possible time sequences of the system mode and controller mode are given in Fig. 1, from which it can be found that the system mode and controller mode are asynchronous. Under the above controller gain matrices, the system state $x(k)$ and control input $u(k)$ are presented in Figs. 2 and 3, respectively, where $x(0)=\left[\begin{array}{lll}-5 & 3 & -2\end{array}\right]^{\mathrm{T}}$ and the exogenous disturbance input $v(k)=\left[\begin{array}{lll}1 / k & 1 / k & 1 / k\end{array}\right]^{\mathrm{T}}$.
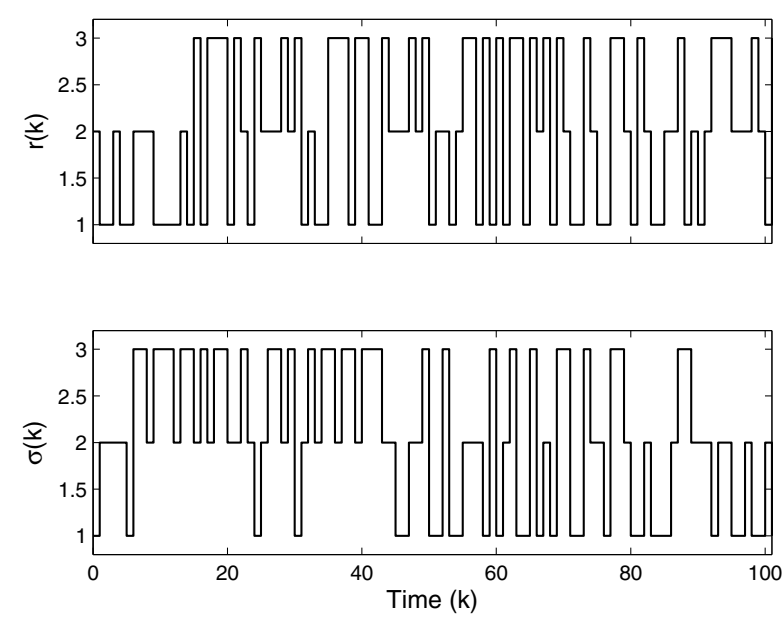

Fig. 1. System and controller modes $r(k)$ and $\sigma(k)$

\section{CONCLusions}

In this paper, the problem of asynchronous control for discrete-time Markov jump systems has been considered. A hidden Markov model has been adopted to describe the asynchronization phenomenon appearing between the system modes and controller modes, which leads to the fact that the closed-loop system is a hidden Markov jump system. Three stochastic passivity conditions have been proposed for the hidden Markov jump system via matrix inequality approach. The existence criterion of the desired asynchronous controller has been developed, which is formulated by LMIs and thus can be solved easily by available LMI toolbox. A numerical example has been given to demonstrate the effectiveness of the proposed design methods. It should be emphasized that because the results of our paper are sufficient conditions only, 


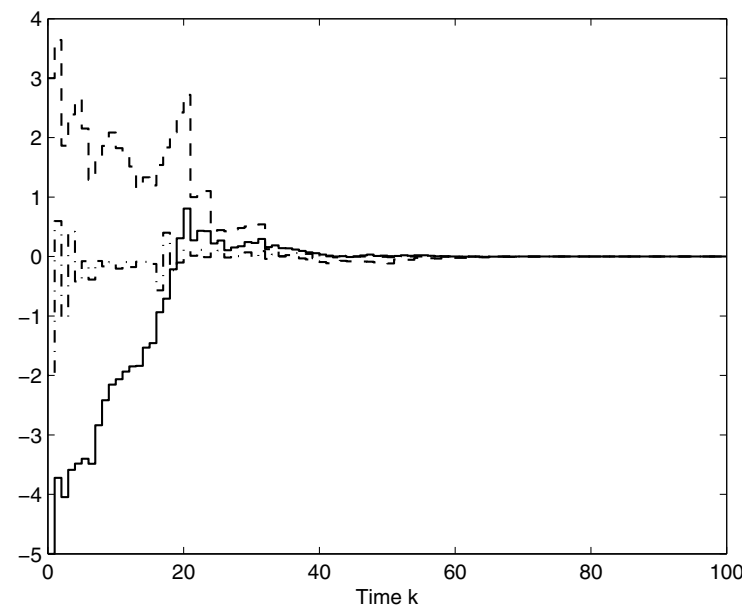

Fig. 2. System state $x(k)$

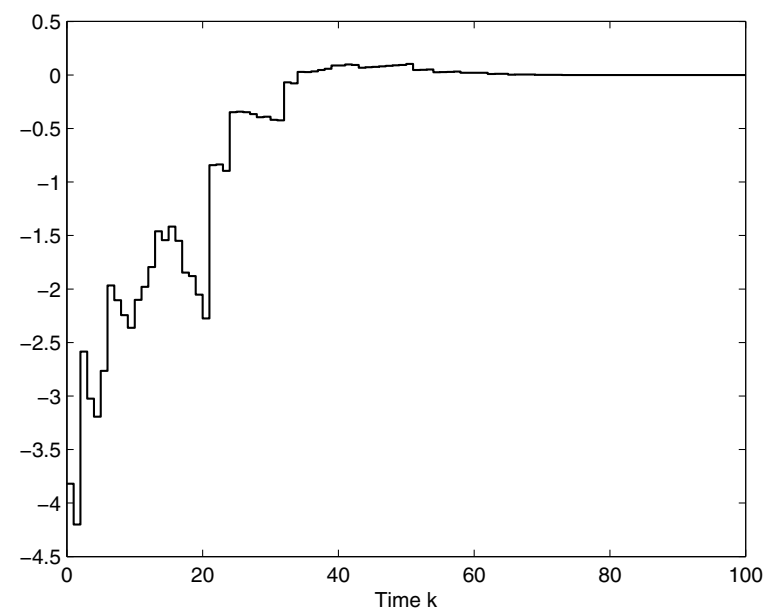

Fig. 3. Control input $u(k)$

there still exists room for improvement. It would be important and more practical to propose novel sufficient conditions with less conservatism or the sufficient and necessary conditions on the passivity analysis of hidden Markov jump systems and the passivity-based asynchronous control for Markov jump systems, which will be one of our future research topics.

\section{REFERENCES}

[1] L. Zhang and J. Lam, "Necessary and sufficient conditions for analysis and synthesis of Markov jump linear systems with incomplete transition descriptions," IEEE Transactions on Automatic Control, vol. 55, pp. 1695-1701, 2010.

[2] L.-S. Hu, P. Shi, and P. M. Frank, "Robust sampled-data control for Markovian jump linear systems," Automatica, vol. 42, pp. 2025-2030, 2006.

[3] P. Bolzern, P. Colaneri, and G. De Nicolao, "Stochastic stability of positive Markov jump linear systems," Automatica, vol. 50, pp. 11811187, 2014.

[4] P. Bolzern, P. Colaneri, and G. De Nicolao, "Almost sure stability of Markov jump linear systems with deterministic switching," IEEE Transactions on Automatic Control, vol. 58, pp. 209-214, 2013.
[5] J. C. Geromel, A. P. C. Gonçalves, and A. R. Fioravanti, "Dynamic output feedback control of discrete-time Markov jump linear systems through linear matrix inequalities," SIAM Journal on Control and Optimization, vol. 48, pp. 573-593, 2009.

[6] P. Bolzern, P. Colaneri, and G. De Nicolao, "Markov jump linear systems with switching transition rates: Mean square stability with dwell-time," Automatica, vol. 46, pp. 1081-1088, 2010

[7] Z. Shu, J. Lam, and S. Xu, "Robust stabilization of Markovian delay systems with delay-dependent exponential estimates," Automatica, vol. 42, pp. 2001-2008, 2006.

[8] Z. Wang, Y. Liu, and X. Liu, "Exponential stabilization of a class of stochastic system with Markovian jump parameters and mode-dependent mixed time-delays," IEEE Transactions on Automatic Control, vol. 55 , pp. 1656-1662, 2010

[9] L. Zhang, E. K. Boukas, and J. Lam, "Analysis and synthesis of Markov jump linear systems with time-varying delays and partially known transition probabilities," IEEE Transactions Automatic Control, vol. 53, pp. 2458-2464, 2008

[10] J. Lam, Z. Shu, S. Xu, and E. K. Boukas, "Robust $H_{\infty}$ control of descriptor discrete-time Markovian jump systems," International Journal of Control, vol. 80, pp. 374-385, 2007.

[11] L. Wu, X. Su, and P. Shi, "Sliding mode control with bounded $\mathscr{L}_{2}$ gain performance of Markovian jump singular time-delay systems," Automatica, vol. 48, pp. 1929-1933, 2012.

[12] H. Dong, Z. Wang, D. W. C. Ho, and H. Gao, "Robust $H_{\infty}$ filtering for markovian jump systems with randomly occurring nonlinearities and sensor saturation: The finite-horizon case," IEEE Transactions on Signal Processing, vol. 59, pp. 3048-3057, 2011.

[13] S. Xu, J. Lam, and X. Mao, "Delay-dependent $H_{\infty}$ control and filtering for uncertain Markovian jump systems with time-varying delays," IEEE Transactions on Circuits and Systems Part I: Regular paper, vol. 54, pp. 2070-2077, 2007.

[14] Z.-G. Wu, P. Shi, H. Su, and J. Chu, " $l_{2}-l_{\infty}$ filter design for discrete-time singular Markovian jump systems with time-varying delays," Information Sciences, vol. 181, pp. 5534-5547, 2011.

[15] J. Xiong and J. Lam, "Stabilization of discrete-time Markovian jump linear systems via time-delayed controllers," Automatica, vol. 42, pp. 747$753,2006$.

[16] C. E. de Souza, "Robust stability and stabilization of uncertain discretetime Markovian jump linear systems," IEEE Transactions on Automatic Control, vol. 51, pp. 836-841, 2006.

[17] C. E. de Souza, A. Trofino, and K. A. Barbosa, "Mode-independent $H_{\infty}$ filters for Markovian jump linear systems," IEEE Transactions on Automatic Control, vol. 51, pp. 1837-1841, 2006.

[18] H. Liu, D. W. C. Ho, and F. Sun, "Design of $H_{\infty}$ filter for Markov jumping linear systems with non-accessible mode information," Automatica, vol. 44, pp. 2655-2660, 2008.

[19] Z. Wu, P. Shi, H. Su, and J. Chu, "Asynchronous $l_{2}-l_{\infty}$ filtering for discrete-time stochastic Markov jump systems with randomly occurred sensor nonlinearities," Automatica, vol. 50, pp. 180-186, 2014.

[20] L. Rabiner, "A tutorial on hidden markov models and selected applications in speech recognition," Proceedings of the IEEE, vol. 77, pp. 257286, 1989.

[21] R. C. L. F. Oliveira, A. N. Vargas, J. B. R. do Val, and P. L. D. Peres, "Mode-independent $\mathrm{H}_{2}$-control of a DC motor modeled as a Markov jump linear system," IEEE Transactions on Control Systems Technology, vol. 22, pp. 1915-1919, 2014. 